Article

\title{
Carbon Nanotube Electron Emitter for X-ray Imaging
}

\author{
Je Hwang Ryu, Jung Su Kang and Kyu Chang Park* \\ Department of Information Display and Advanced Display Research Center, Kyung Hee University, \\ Seoul 130-701, Korea; E-Mail: jyoo@khu.ac.kr (J.H.R.); kangjungsu@khu.ac.kr (J.S.K) \\ * Author to whom correspondence should be addressed; E-Mail: kyupark@khu.ac.kr; \\ Tel.: +82-2-961-9447; Fax: +82-2-968-6924.
}

Received: 21 September 2012; in revised form: 24 October 2012 / Accepted: 12 November 2012 / Published: 16 November 2012

\begin{abstract}
The carbon nanotube field emitter array was grown on silicon substrate through a resist-assisted patterning (RAP) process. The shape of the carbon nanotube array is elliptical with $2.0 \times 0.5 \mathrm{~mm}^{2}$ for an isotropic focal spot size at anode target. The field emission properties with triode electrodes show a gate turn-on field of $3 \mathrm{~V} / \mu \mathrm{m}$ at an anode emission current of $0.1 \mathrm{~mA}$. The author demonstrated the X-ray source with triode electrode structure utilizing the carbon nanotube emitter, and the transmitted X-ray image was of high resolution.
\end{abstract}

Keywords: carbon nanotube; X-ray; resist assisted patterning

\section{Introduction}

Carbon nanotubes (CNT) show excellent properties that make them attractive for several nanoelectronic device applications, including electron emitters [1]. CNTs exhibit characteristics, such as high aspect ratio, high thermal conductivity, and low chemical reactivity, which also make them suitable for the functionalized fabrication of X-ray tubes, and X-ray fluorescence (XRF), X-ray diffraction (XRD) [2,3]. Stable field emission is the key requirement for CNTs in high performance electron emission source applications [4]. Thus, there is a critical need for novel methods for growing CNTs. Electron sources for X-ray generation in tomosynthesis require an anode current that is greater than $100 \mathrm{~mA}$ and endures high anode voltage $[5,6]$.

Conventional CNT emitters such as electrophoresis process, chemical vapor deposition, and printing method process, do not meet this requirement because they quickly degrade during high 
current operation. Also, emission current is not enough for those devices. Stable and reliable electron emission is also necessary for extended operation. For enhanced and stabilized electron emission, electron sources must strongly adhere to the substrate, be robust under environmental stress, and possess sharp tips, which do not change and degrade during operation.

In this paper, we describe the growth and post-treatments of CNTs for making high-performance emitters. The CNT emitter grown using the RAP process can prevent chemical reactions of carbon with gases in the environment and improve the stability of the CNTs. Also, by using the CNT emitter, we demonstrated the proto-type X-ray imaging system based on CNT emitter. The structural and electron emission properties of the emitters and their application of X-ray imaging are discussed.

\section{Experimental Section}

\subsection{Growth of Carbon Nanotube for High-Performance Emitters}

The CNTs were grown using a resist-assisted patterning (RAP) process, which has been reported in detail elsewhere [7]. For the RAP process, silicon substrates were used to grow the CNTs in Figure 1a. As shown the Figure 1b, the nickel catalyst layer with thickness of $10 \mathrm{~nm}$ was deposited on the silicon substrate using radio frequency magnetron sputtering. The nickel catalyst layer was patterned using photo-resist. The resist was patterned elliptical shape of $2.0 \times 0.5 \mathrm{~mm}$, and inner pattern is $3 \mu \mathrm{m}$ sized islands with an island-to-island pitch of $15 \mu \mathrm{m}$. The $15 \mu \mathrm{m}$ pitch is designed to reduce field screening by nearest emitters. The resist was not removed for seed formation and is required for CNT growth. The unwanted nickel catalyst was etched, and then the substrate was annealed in a high-temperature furnace in Figure 1c. The annealing temperature and time in this study were $600{ }^{\circ} \mathrm{C}$ and $30 \mathrm{~min}$, respectively. After annealing, the nickel catalyst separated into small grains for the growth of the CNTs. CNTs can grow where there is carbon-network formation after the annealing process. In Figure 1d, the CNTs were grown in a triode direct current-plasma enhanced chemical vapor deposition dc-PECVD system with a mesh grid placed $10 \mathrm{~mm}$ above the substrate holder electrode. With this triode dc-PECVD system, the growth of CNTs is easily enhanced and inhibited by strong positive and negative bias voltages on the mesh electrode, respectively [8].

Figure 1. Process flow of carbon nanotubes (CNT) emitters for X-ray source. (a) Cleaned Si substrate; (b) Ni catalyst deposition; (c) Patterning and forming for seed formation; (d) CNT growth; (e) hydrofluoric (HF) treatment; (f) Electrical aging treatment.

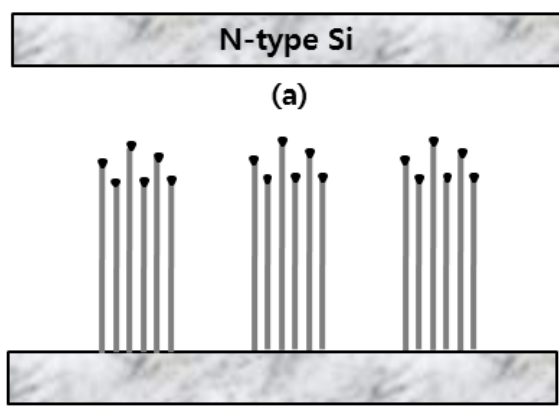

(d)

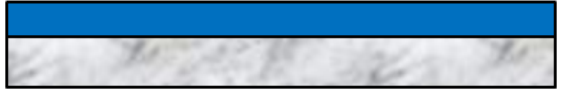

(b)

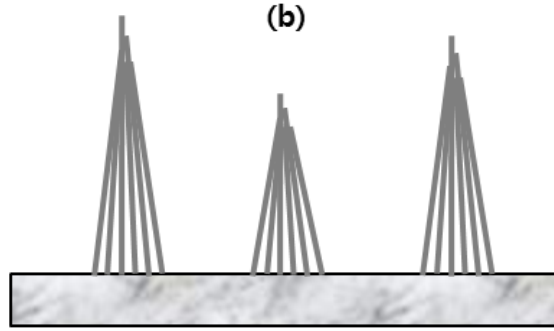

(e)

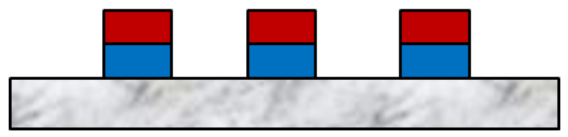

(c)

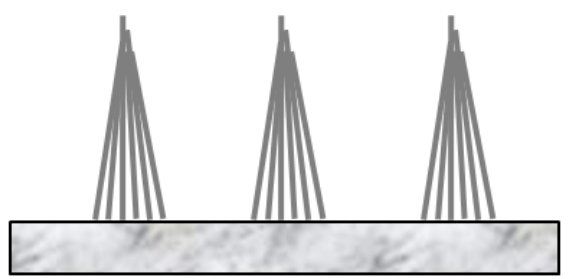

(f) 
The CNT density and length can be changed easily by changing the mesh electrode bias and polarity. The mesh electrode bias voltage can alter the CNT growth mechanism by modifying the flux of the ions impinging on the substrate. A positive mesh bias increases the ion flux and electric field, resulting in increased CNT length and density the higher the bias, the higher the length and density. However, a negative mesh bias, resulting in a reduced ion flux, reduces CNT density and growth rate probably because of catalyst particle poisoning by an amorphous carbon deposit that cannot be removed through sputtering.

After growing the patterned CNT emitters of elliptical shape in Figure 1d, they were performed the post-treatment with hydrofluoric (HF) acid. The interface between the CNTs and substrate was robust enough to withstand HF treatment. After HF treatment, CNT emitters were get-together in the tip position, resulting cone-shape formation. The cone shape formation could enhance stability of electron emission current. The HF treatment is a very simple and cost-effective process for improving the electronic properties of CNTs emitter [9].

And then, we carried the 2 nd post treatment which the effect of electrical aging in high vacuum chamber. By the electrical aging treatment, the nickel catalysts were removed from top of nanotube, and the electron emission current was enhanced and the emission stability was improved for high-performance X-ray source. Finally, we can obtain the sharpened and uniform emitters [10].

The structural properties of the CNTs were characterized through field emission scanning electron microscopy (FE-SEM) and transmission electron microscopy (TEM).

\subsection{X-ray Generation with the High-Performance CNT-Emitters}

The X-rays were generated with a four electrodes X-ray system including electrodes with anode, focusing, gate, and cathode. The focal spot size of X-ray generation was found to depend on the emitter arrays geometry. Figure $2 \mathrm{a}$ shows the schematic diagram of the X-ray generation system. The electrons were extracted from grounded CNT emitters by controlling the gate electrode, and became focused electron with adjusting focus voltage. The focused electrons were rapidly decelerated by the anode target to produce characteristic X-rays. The distance between anode with a molybdenum and gate was maintained at $8 \mathrm{~mm}$. The base pressure of the X-ray generation system was kept at $1 \times 10^{-7}$ Torr.

Figure 2. (a) Schematic diagram and (b) a photo image of the X-ray generator with CNT emitter.

(a)

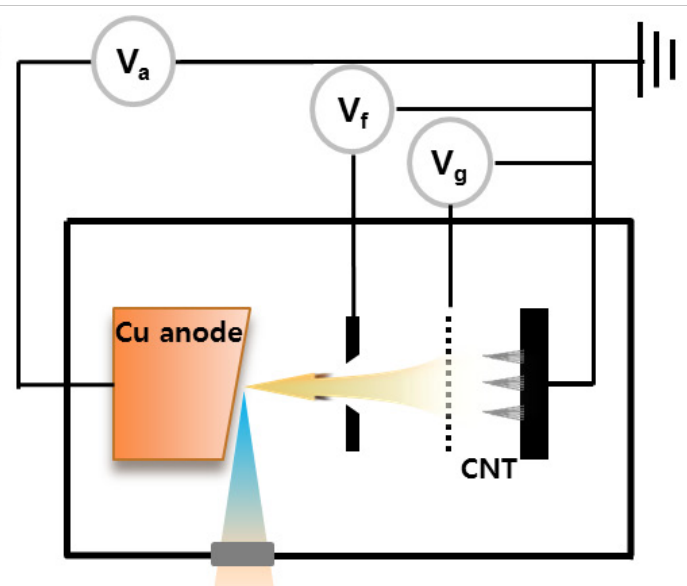

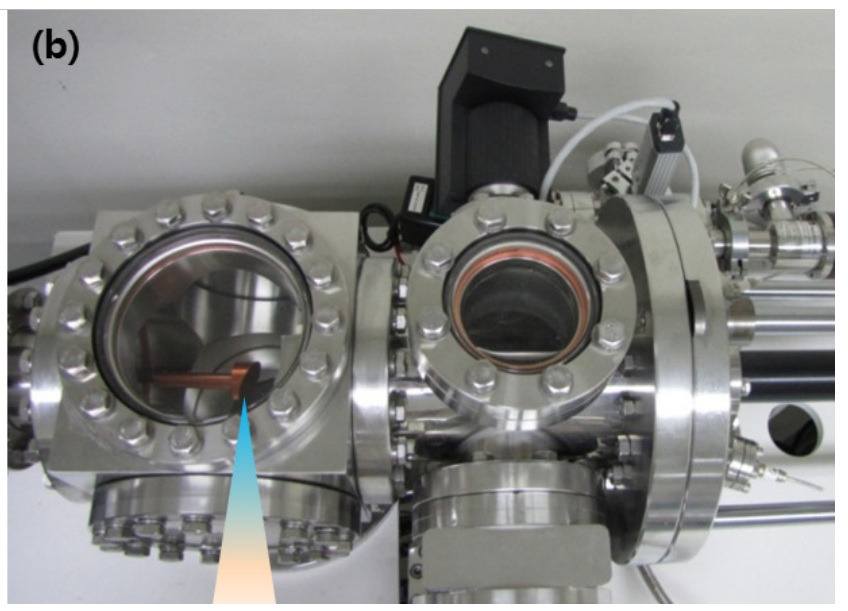


Figure $2 \mathrm{~b}$ shows the photograph image of triode type X-ray system. In these experiments, we use vacuum system for $\mathrm{X}$-ray generation instead of vacuum sealing tube. The vacuum sealing technique for colt cathode is not matured. The full size of the system $20 \mathrm{~cm} \times 20 \mathrm{~cm} \times 50 \mathrm{~cm}(\mathrm{H} \times \mathrm{V} \times \mathrm{W})$ and sample was loaded through load-lock chamber and X-ray generation chamber keep ultra-high pressure. The CNT electron source was delivered through home-made delivery systems. The X-ray system design utilizing CNT cathode may be a significant advance in X-ray technology development and could lead to portable and miniature X-ray sources for medical and industrial applications.

\section{Results}

\subsection{CNT Emitters for High-Performance X-ray}

For the isotropic focal spot at anode target, the emitter is an elliptical area of $2.0 \mathrm{~mm} \times 0.5 \mathrm{~mm}$ as shown in Figure 3a [11]. The initial CNTs without post-treatment are vertically aligned and formed several nickel catalysts on top of multiwall CNTs [7]. However, after post-treatments, there is no nickel catalyst. A SEM image of the vertically aligned CNTs is shown in Figure 3b. One CNTs bundle had a diameter of $3 \mu \mathrm{m}$, and they were precisely pitched every $15 \mu \mathrm{m}$. The island size and pitch of the CNTs could be controlled through the catalyst fabrication process as shown in Figure 1c. The magnified image of CNTs is shown in Figure 3c.

Figure 3. SEM images of the (a) elliptical emitter with $2.0 \mathrm{~mm} \times 0.5 \mathrm{~mm}$ formed by RAP process; (b) magnified image of CNT array emitters with $3 \mu \mathrm{m}$ islands and $15 \mu \mathrm{m}$ pitch; (c) a magnified image of one CNT-bundles.

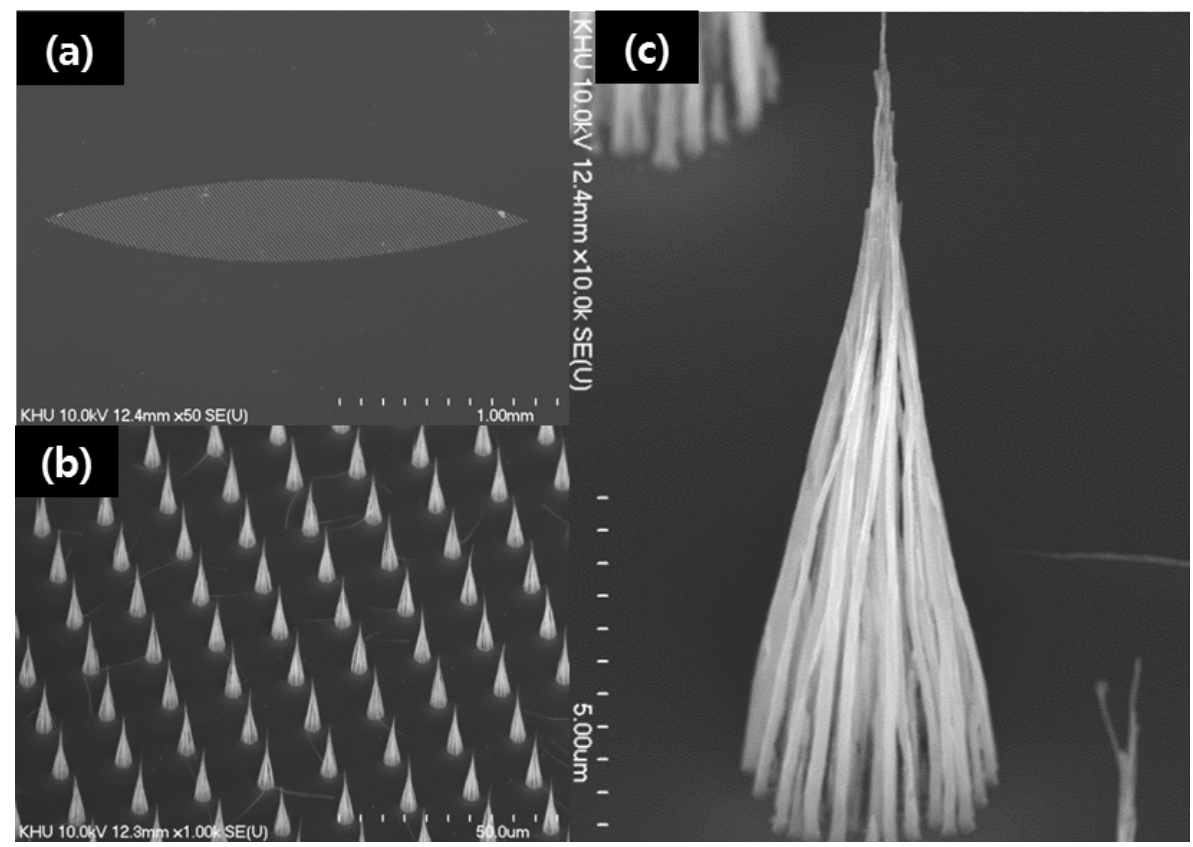

The figure clearly shows that the CNT had a sharp tip and a wide bottom. It was vertically aligned and well positioned in the predefined island area. The length of the CNT was over $10 \mu \mathrm{m}$. The one CNT-bundle emitter was cone shaped. The tip apex measured a few nanometers in diameter, and the diameter of the bottom region in contact with the substrate was equal to the pre-patterned dot area of 
the $3 \mu \mathrm{m}$ island. The adhesion of conventional single CNTs onto the substrate is weak [12]. This is thought to increase the adhesion between the CNTs and the substrate, also to reduce the screening effect between emitters and adjacent emitter [13].

As shown in Figure 4, the diameter and angle of the tip were in the nanometer range and around $25.30^{\circ}$, respectively. The emitter tip was cone shaped with a sharp tip. The tip apex measured a few nanometers in diameter. There was one CNT-bundle emitter with multiple rods in the bottom. To understand the structural properties, Additional details concerning the mechanism can be found in a previously published report [12].

Figure 4. TEM image of a magnified image of the tip apex of a CNT emitter.

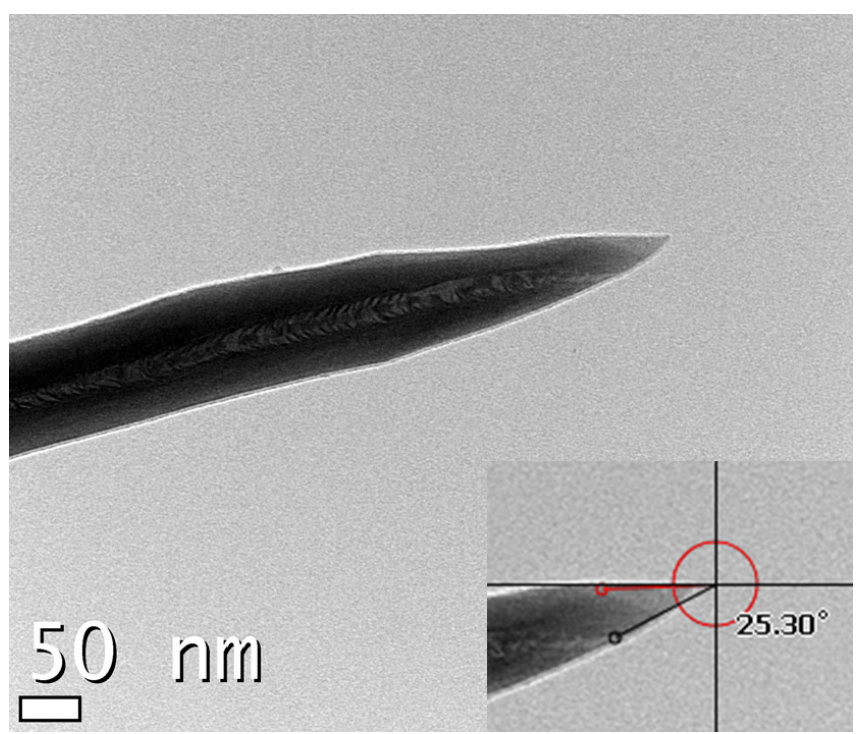

\subsection{X-ray Generation with the High-Performance CNT-Emitters}

Figure 5 shows the electron field emission properties of the elliptical CNT emitters. Prior to obtaining X-ray imaging, we performed the field emission measurements on the diode structure. Through the diode structure, we can establish the material field emission properties without module problems, such as anode melting and arcing effect between gate and cathode. The obtained emission current on direct current mode is $90 \mathrm{~mA}$ at $2100 \mathrm{~V}(7.5 \mathrm{~V} / \mu \mathrm{m})$. For X-ray imaging, the emission current was measured using a four electrodes structure as shown in Figure 2. The acceleration voltage was applied to anode of $50 \mathrm{kV}$. The cathode current of $0.5 \mathrm{~mA}$ was obtained at a gate electric field of $4 \mathrm{~V} / \mu \mathrm{m}$. The anode current was measured by adjusting the gate electrode in the dc mode. The transmission rate to anode target was $\sim 70 \%$ from elliptical emitter.

Figure 6 shows X-ray images of dynamic random-access memory and the biological object. The electrons were extracted from the emitter by the positive gate field $4 \mathrm{~V} / \mu \mathrm{m}$ applied to the gate electrode. For X-ray generation, the anode voltage and current was maintained at $50 \mathrm{kV}$ and $0.5 \mathrm{~mA}$, respectively, with an exposure time of $3 \mathrm{~s}$. We can clearly see the transmitted-ray images of various objects. Based on this research, in future we will apply more emphasis to X-ray properties of our CNT electron based X-ray generation. 
Figure 5. Field emission properties of (a) diode and (b) triode measurement for X-ray imaging.
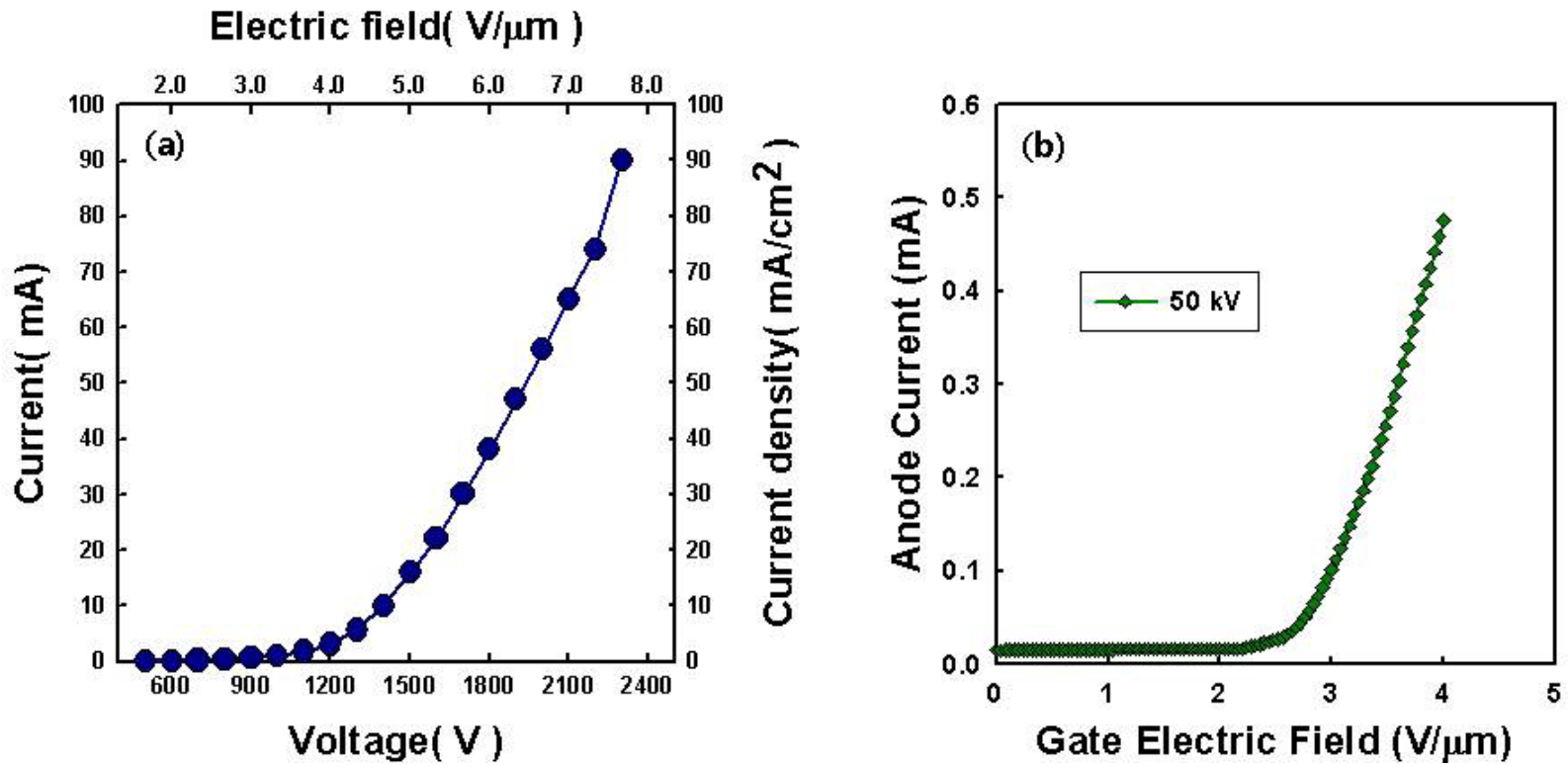

Figure 6. X-ray images of (a) dynamic random-access memory; (b) chest region of mouse.

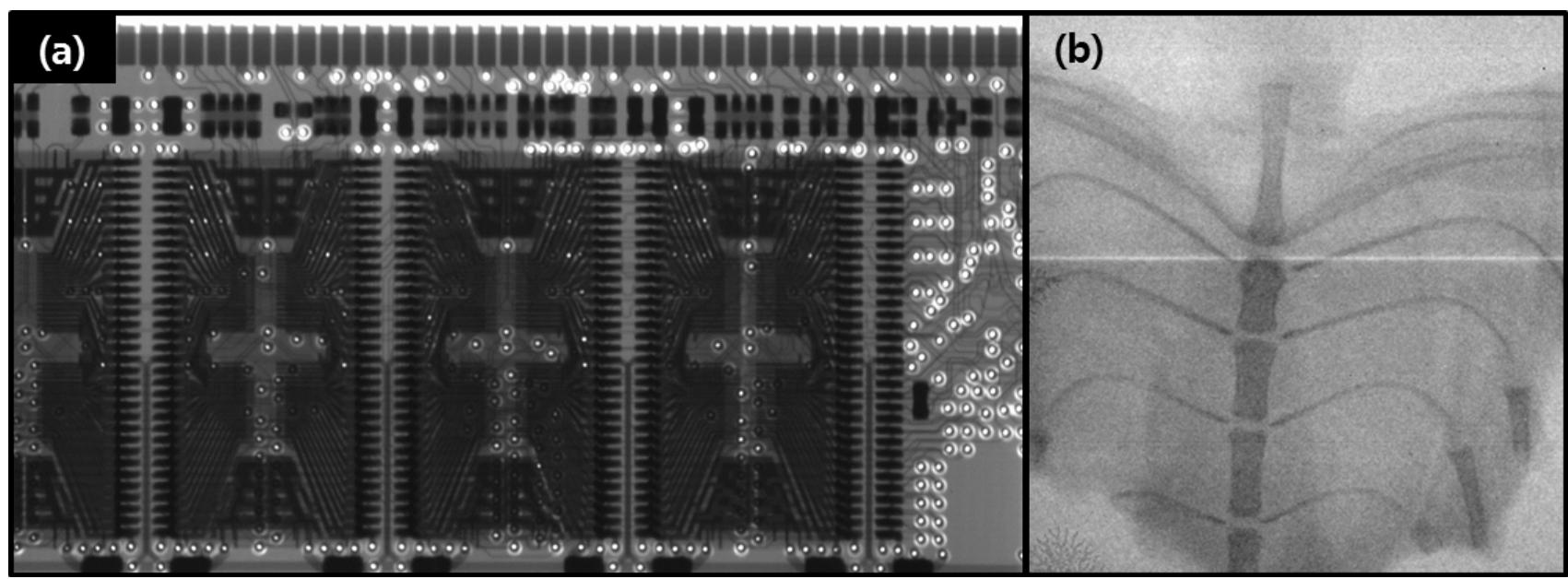

\section{Conclusions}

A CNT based field emission X-ray source with four electrodes including anode, focusing, gate, and cathode has been demonstrated. The high-performance CNT emitter grown using the RAP process appears to be an excellent candidate for electron sources for X-ray generation. The CNT-bundles emitters of elliptical shape were fabricated using the RAP with lithography process. In the post-treatment process with HF dipping and electrical aging, we could then modify emitters to align the cone shape. The cone shape emitters produced an enhanced and stable electron emission. This enhanced emission combined with the ability to control emitter pitch, produces an enhancement of the X-ray image. With these emitters, we obtained high resolution X-ray images of on industrial product and a small animal. 


\section{Acknowledgments}

This work was supported by the National Research Foundation of Korea (NRF) grant funded by the Korea government (MEST) (No. 2011-0021475).

\section{References}

1. Baughman, R.H. Carbon nanotubes - The route toward applications. Science 2010, 297, 787-792.

2. Wang, S.; Calderon, X.; Peng, R.; Schreiber, E.C.; Zhou, O.; Chang, S. A carbon nanotube field emission multipixel X-ray array source for microradiotherapy application. Appl. Phys. Lett. 2011, 98, 1-4.

3. van der Snickt, G.; Janssens, K.H.; Dik, J.; de Nolf, W.; Vanmeert, F.; Jaroszewicz, J.; Cotte, M.; Falkenberg, G.; van der Loeff, L. Combined use of synchrotron radiation-based $\mu-X R F, \mu-X R D$, $\mu$-XANES and $\mu$-FTIR reveals an alternative degradation pathway of the pigment cadmium yellow (CdS) in a painting by Van Gogh. Anal. Chem. 2012, doi:10.1021/ac3015627.

4. Minoux, E.; Groening, O.; Teo, K.B.; Dalal, S.H.; Gangloff, L.; Schnell, J.P.; Hudanski, L.; Bu, I.Y.; Vincent, P.; Legagneux, P.; Amaratunga, G.A.; Milne, W.I. Achieving high-current carbon nanotube emitters. Nano Lett. 2005, 5, 2135-2138.

5. Ascenti, G.; Krauss, B.; Mazziotti, S.; Mileto, A.; Settineri, N.; Vinci, S.; Donato, R.; Gaeta, M. Dual-energy computed tomography (DECT) in renal masses. Acad. Radiol. 2012, 19, 1-8.

6. Jung, J.W.; Lee, J.S.; Kwon, S.J.; Park, S.J.; Chang, S.; Kim, J.; Pyo, J.; Kohmura, Y.; Nishino, Y.; Yamamoto, M.; Ishikawa, T.; Je, J.H. Fast microtomography using bright monochromatic X-rays. Rev. Sci. Instrum. 2012, 83, 093704:1-093704:5.

7. Park, K.; Ryu, J.; Kim, K.; Yu, Y. Growth of carbon nanotubes with resist-assisted patterning process. J. Vac. Sci. Technol. B 2007, 25, 1261-1264.

8. Park, K.; Jang, J.; Ryu, J. Field Emission Display and Manufacturing Method of the Same Having Selective Array of Electron Emission Source. US Patent 8, 172,633, 8 May 2012.

9. Kim, K.S.; Ryu, J.H.; Lee, C.S.; Jang, J.; Park, K.C. Enhanced and stable electron emission of carbon nanotube emitter arrays by post-growth hydrofluoric acid treatment. J. Mater. Sci. 2009, 20, 120-124.

10. Ryu, J.H.; Kim, K.S.; Lee, C.S.; Jang, J.; Park, K.C. Effect of electrical aging on field emission from carbon nanotube field emitter arrays. J. Vac. Sci. Technol. B 2008, 26, 856-859.

11. Liu, Z.; Yang, G.; Lee, Y.Z.; Bordelon, D.; Lu, J.; Zhou, O. Carbon nanotube based microfocus field emission X-ray source for microcomputed tomography. Appl. Phys. Lett. 2006, 89, 103-111.

12. Ryu, J.H.; Bae, N.Y.; Oh, H.M.; Zhou, O.; Jang, J.; Park, K.C. Stabilized electron emission from silicon coated carbon nanotubes for a high-performance electron source. J. Vac. Sci. Technol. B 2011, 29, 02B120:1-02B120:5.

13. Nilsson, L.; Groening, O.; Emmenegger, C.; Kuettel, O.; Schaller, E.; Schlapbach, L.; Kind, H.; Bonard, J.; Kern, K. Scanning field emission from patterned carbon nanotube films. Appl. Phys. Lett. 2000, 76, 2071-2073.

(C) 2012 by the authors; licensee MDPI, Basel, Switzerland. This article is an open access article distributed under the terms and conditions of the Creative Commons Attribution license (http://creativecommons.org/licenses/by/3.0/). 